\title{
Hepatocellular Carcinomas Show Abnormal Expression of Fibronectin Protein
}

Michael Torbenson, M.D., Jianzhou Wang, M.D., Michael Choti, M.D., Raheela Ashfaq, M.D., Anirban Maitra, M.D., Robb E. Wilentz, M.D., John Boitnott, M.D.

Departments of Pathology (MT, AM, REW, JB) and Surgery (MC), The Johns Hopkins Hospital, Baltimore, Maryland; Department of Pathology, University of Oklahoma Health Sciences Center (JW), Oklahoma City, Oklahoma; and Department of Pathology, University of Texas Southwestern Medical Center (RA), Dallas, Texas

Fibronectin plays an important role in cell-to-cell adhesion, cell migration, and cell signaling. In the liver, fibronectin expression has been studied primarily as a component of the extracellular matrix, but little information is available on the expression of fibronectin protein in the neoplastic cells of hepatocellular carcinomas (HCCs). Twenty-four surgically resected HCCs were immunostained with fibronectin. Tumor and normal liver tissues were concurrently analyzed in all cases, and expression in the tumor was evaluated in comparison to the nonneoplastic liver. The average age at resection was $54 \pm 18$ years for the 18 men and 6 women. Twenty-one of the cases were classic HCCs including 6 cases that were well differentiated, 12 cases moderately differentiated, and 3 cases poorly differentiated. The remaining 3 cases were moderately differentiated fibrolamellar carcinomas. In the normal liver, fibronectin labeled the sinusoids and weakly to moderately stained the cytoplasm of hepatocytes. In HCCs, 15/24 showed overexpression of fibronectin in the cytoplasm, 8/24 showed no change from the nonneoplastic liver, and one case showed decreased cytoplasmic staining. In addition, an abnormal membranous staining pattern was noted in 16/24 HCCs. In contrast to the HCCs, none of the three fibrolamellar carcinomas showed increased cytoplasmic or membranous staining. Excluding fibrolamellar carcinoma, increased cytoplasmic staining and/or an abnormal membranous staining was noted in 19/21 (90\%) of HCCs. Fi-

Copyright (C) 2002 by The United States and Canadian Academy of Pathology, Inc.

VOL. 15, NO. 8, P. 826, 2002 Printed in the U.S.A.

Date of acceptance: April 16, 2002.

Supported in part by the Margaret Lee Fund for Hepatopancreatobiliary Research.

Address reprint requests to: Michael Torbenson, M.D., Department of Pathology, Ross Building, Room 632, The Johns Hopkins University School of Medicine, 720 Rutland Avenue, Baltimore, MD 21205-2196; e-mail: mtorben@jhmi.edu; fax: 410-614-9011.

DOI: 10.1097/01.MP.0000024257.83046.7C bronectin shows abnormal cytoplasmic and/or membranous staining in the majority of HCCs. The implications of fibronectin overexpression are uncertain but may reflect a critical step in tumor genesis.

KEY WORDS: Fibrolamellar carcinoma, Fibronectin, Hepatocellular carcinoma, Immunohistochemistry.

Mod Pathol 2002;15(8):826-830

Fibronectin is a high molecular weight glycoprotein that can bind a number of extracellular and cellular components including collagen, glycosaminoglycans, actin, and DNA (1). Fibronectin is important in a wide variety of fundamental cell processes including cell-to-cell adhesion, cell-to-extracellular matrix adhesion, and cell migration (1). Fibronectin is also found in the plasma, where it is involved in blood clot formation and opsonization $(2,3)$. This functional diversity of the fibronectin gene results from alternative splicing of pre-mRNA, leading to multiple protein isoforms with unique biological properties $(4,5)$.

Fibronectin plays an important role in signal transduction and cell adhesion and is partially regulated by TGF- $\beta$ (6), a cytokine that plays a central role in controlling the growth of normal hepatocytes. Through interactions with integrins, fibronectin can also directly influence gene transcription and cellular morphology in hepatocytes (7). We hypothesized that abnormalities of fibronectin expression may play an important role in hepatocellular carcinoma (HCC) tumor biology. Although fibronectin has been studied in relationship to fibrosis $(8,9)$, its role in HCC has been incompletely characterized. Previous studies have consistently demonstrated increased transcription of fibronectin including abnormal splice variants (1013), but protein expression results have been inconsistent $(10,14,15)$. Thus, we examined the 
expression of fibronectin protein in HCC by immunohistochemistry. Immunohistochemistry provides the advantage of precise cellular localization, which is important because cellular forms of fibronectin can be synthesized by a number of nonhepatocyte cell types residing in the liver, including endothelial cells (1), stellate cells (16), and macrophages (17).

\section{MATERIALS AND METHODS}

\section{Case Selection}

A retrospective review of the surgical pathology files of the Johns Hopkins Department of Pathology revealed 24 cases of HCC with available paraffin blocks. All specimens were from partial hepatectomy surgeries performed between 1991 and 2001 . Histological sections were obtained from formalinfixed, paraffin-embedded tissues, and all cases were retrospectively reviewed by one pathologist (MST) to confirm the diagnosis of HCC. For immunohistochemistry, a representative block was chosen that contained tumor and adjacent nonneoplastic liver.

\section{Immunohistochemistry}

Immunostaining was performed at room temperature and carried out on a DAKO Autostainer (DAKO, Carpinteria, CA). Reagents were used as supplied in the Envision Plus Detection Kit (DAKO). A polyclonal rabbit anti-human fibronectin antibody was used (A0245, DAKO) at a dilution of 1:1200. A known positive control section was included in each run to assure proper staining.

Paraffin sections were cut at $5 \mu \mathrm{m}$, deparaffinized, and placed in $200 \mathrm{~mL}$ of DAKO Target Retrieval Solution, $\mathrm{pH}$ 6.0. The buffer was brought to a temperature of $100^{\circ} \mathrm{C}$ for 20 minutes. Sections were then removed and allowed to cool in buffer for 20 minutes and were rinsed thoroughly in deionized water. Sections were then quenched with $3 \% \mathrm{H}_{2} \mathrm{O} 2$ for 5 minutes and incubated with the primary antibody for 30 minutes. After primary antibody, the sections were incubated for 30 minutes in DAKO EnVision + Peroxidase, Rabbit or Mouse, a labeleddextran polymer. Sections were then incubated in a freshly prepared mixture of diaminobenzidine (3,3'-diaminobenzidine) and buffer substrate solution for 5 minutes. Finally, sections were counterstained with hematoxylin and blued in Richard Allen Bluing Reagent, dehydrated in a graded series of ethanols and xylene, and coverslipped. Negative controls were included in each immunostain run.

The fibronectin staining in the tumors was evaluated in relationship to the concurrently analyzed nonneoplastic liver, and cytoplasmic staining was scored as decreased, similar, or increased from nonneoplastic liver. The intensity of staining for normal liver and tumor was also scored as mild $(1+)$, moderate $(2+)$, and marked $(3+)$. The presence of sinusoidal staining and membranous staining was also noted.

\section{RESULTS}

The mean age at resection was $54 \pm 18$ years for the 18 men and 6 women with HCCs. Eighteen patients were Caucasian, 3 African-American, 2 Arab-American, and 1 Hispanic-American. There was no significant difference in age at resection for the men and women $(P=0.07)$. Six cases were well differentiated, 12 cases moderately differentiated, and 3 cases poorly differentiated. Sixteen of the cases were typical HCCs, 2 cases were clear-cell HCC, and the remaining 3 cases were fibrolamellar carcinomas. Fourteen cases arose in the background of chronic hepatitis $C$ viral infection $(n=8)$, chronic hepatitis B viral infection (3), hepatitis B and C coinfection (1), cryptogenic cirrhosis (1), and hereditary hemochromatosis (1). Seven HCCs arose in noncirrhotic livers in patients with no known underlying liver disease, as did the three fibrolamellar carcinomas.

In the normal liver, antibodies to fibronectin labeled the sinusoids and weakly $(20$ cases, $1+)$ to moderately (4 cases, $2+$ ) stained the cytoplasm of hepatocytes (Fig. 1A). Staining was also seen in fibrotic portal tracts, particularly in fibrotic portal tracts near the tumor, though nonfibrotic tracts were negative. Ścattered endothelial cells were also positive, as were macrophages and cells located in the lobules morphologically consistent with Kupffer cells.

In HCCs, 15/24 demonstrated cytoplasmic overexpression of fibronectin (8 cases, $2+$; 7 cases, $3+$ ) compared with the adjacent nonneoplastic liver (Fig. 1B), 8/24 showed no change, and 1 case showed decreased cytoplasmic staining. In addition, an abnormal, dense membranous staining pattern was noted in 16/24 HCCs and was characterized by a heavy membranelike pattern of staining around the entire circumference of each individual cell (Fig. 1C). This pattern was distinct from sinusoidal staining, which was also present in the tumors. Overall, abnormal staining in HCC fell into the following categories: increased cytoplasmic and membranous staining ( $n=12)$; increased cytoplasmic without membranous staining $(n=3)$; no increased cytoplasmic staining, but membranous staining $(n=3)$; and decreased cytoplasmic staining, but membranous staining $(n=1)$. The tumor capsules were also positive for fibronectin (Fig. 1D). No differences in staining were noted for HCCs arising in cirrhotic versus noncirrhotic livers. No correlation between gender, race, or degree of tu- 
mor differentiation was noted for any of the staining patterns. No nuclear staining was seen in any cases.

In addition to the above staining patterns, one additional pattern was noted, where scattered single hepatocytes were more brightly positive than adjacent cells (Fig. 1E). This pattern was noted in $18 / 24$ of the HCCs and 21/24 of the non-neoplastic livers. These more brightly positive hepatocytes were located in all portions of the lobules with no clear geographical localization and were morphologically identical to neighboring hepatocytes. Their density varied from rare cells to approximately $5 \%$ of hepatocytes.

In contrast to the HCCs, none of the three fibrolamellar carcinomas showed increased cytoplasmic or membranous staining (Fig. 1F). In a single case of fibrolamellar carcinoma, the nonneoplastic liver showed focal membranous staining. Thus, excluding the fibrolamellar carcinomas, increased cyto-


FIGURE 1. Fibronectin is normally expressed in hepatocytes with a weak $(1+)$ diffuse cytoplasmic staining pattern and a sinusoidal staining pattern (A). Fibronectin in a hepatocellular carcinoma (HCC) shows increased cytoplasmic staining ( $3+$ ) in comparison to adjacent nonneoplastic liver (B). Abnormal membranous pattern of staining is also seen in HCC (C). The capsules of the tumors stained for fibronectin (D). In both normal liver and tumor (inset), occasional scattered hepatocytes were more brightly positive than neighboring cells (E). In contrast, fibrolamellar carcinomas failed to show increased cytoplasmic or membranous staining $(\mathbf{F})$. 
plasmic staining, and/or an abnormal membranous staining was noted in 19/21 (90\%) of HCCs. In the two cases of typical HCC that showed no abnormalities of fibronectin expression, both were morphologically typical well-differentiated HCCs.

\section{DISCUSSION}

Fibronectin proteins can be divided into insoluble cellular fibronectin and soluble plasma fibronectin. Cellular fibronectin is important in wound healing and scar formation in the dermis (18) and plays a role in the scarring that characterizes liver cirrhosis $(8,9)$. Plasma fibronectin, which is produced primarily in hepatocytes, plays an important role in clot formation (19), opsonization of bacteria (3), and opsonization of circulating collagenous degradation products (2). The antibody used in this study was raised against a plasma-derived immunogen, but it appears to recognize both cellular and plasma forms of fibronectin based on the staining of nonhepatocyte cell types (endothelial cells, Kupffer cells, macrophages) that are known to produce cellular fibronectin but are not known to produce plasma fibronectin $(1,17)$.

In this study, fibronectin protein showed abnormal expression in $90 \%$ of the nonfibrolamellar HCCs with increased cytoplasmic staining and/or abnormal membranous staining compared with adjacent non-neoplastic liver. The presence of abnormal protein expression in such a high percentage of cases suggests a potentially important role for fibronectin in HCC tumor biology. Overexpression of fibronectin has also been reported in hepatoblastomas (20). Interestingly, abnormal expression of fibronectin has been reported in primate (21), rodent (22), and avian HCCs (23) as well. These findings suggest an important and "conserved" function for this protein in HCCs. One possibility in this regard is control of G1 cell cycle progression, which is actively regulated by extracellular fibronectin (24). Abnormal fibronectin protein expression in HCCs may be a manifestation of dysregulation of this important cell cycle control point. Furthermore, fibronectin either regulates, or is regulated by, a number of important cell cycle proteins thought to play a role in HCC pathogenesis including cyclin D1 (24), p53 (25), and $\beta$-catenin (24). Abnormal fibronectin expression may also lead to abnormal cell-to-cell or cell-to-extracellular matrix adhesion, contributing to tumor development. In contrast to the classic HCC, the fibrolamellar variants did not show fibronectin expression, though the numbers of cases in this study are too small to draw firm conclusions.

Overexpression of fibronectin protein in human HCCs has been investigated in a small number of previous reports. In a Japanese report on fibronectin overexpression in thyroid carcinomas, 13 HCCs were also studied and only 4 (31\%) showed increased cytoplasmic fibronectin labeling by immunohistochemistry (15). However, these investigators also reported that the normal hepatocytes stained in only a minority of their cases, in contrast to the findings in this study in which all of the cases showed at least weak expression in the nonneoplastic liver. The reasons for these discrepancies are uncertain, but differences in staining techniques may have played a role. Other previous studies have emphasized increased sinusoidal staining of fibronectin in HCCs $(14,26,27)$, but they also reported cytoplasmic and membranous staining in some cases $(14,26)$. At the ultrastructural level, cytoplasmic fibronectin has been demonstrated by routine electron microscopy and by immunoelectron microscopy in neoplastic cells of HCC (28). Increased mRNA and abnormal splice variants in HCC have also been reported by a number of authors (10-13). Taken together, the results from our study and the literature demonstrate abnormal expression of fibronectin in HCC at the mRNA and protein levels. Our study significantly extends the few previous studies that reported immunohistochemical findings by demonstrating that almost all non-fibrolamellar HCCs show abnormal patterns of fibronectin expression, with either increased cytoplasmic and/or membranous staining.

Finally, a third pattern of staining for fibronectin was also noted in this study, with scattered single hepatocytes staining more brightly for fibronectin than neighboring hepatocytes. This pattern is somewhat reminiscent of early reports of immunostaining for albumin $(29,30)$, which also showed scattered positive cells and initially suggested the possibility of functional diversity in production of albumin by hepatocytes, with overexpression in some cells allowing other hepatocytes to more actively produce other needed proteins. However, subsequent studies did not confirm this hypothesis (31). The significance of this fibronectin-staining pattern in these cases is uncertain.

The overexpression of fibronectin seen in this study adds to our understanding of HCC tumor biology, but it also may have implications for routine surgical pathology diagnostics. Although this possibility was not addressed in this study, as all of the cases were typical HCCs and presented no diagnostic dilemmas, the high percentage of cases with fibronectin overexpression suggests it may be a useful antibody to include in further studies that develop immunostain panels to evaluate difficult tumors of the liver.

In conclusion, in addition to its important role in the extracellular matrix, fibronectin shows abnormal cytoplasmic and/or membranous staining in 
the majority of HCCs, with $90 \%$ of the cases in this study showing abnormal protein expression. In contrast, the few fibrolamellar carcinomas included in this study failed to show abnormal fibronectin expression in the tumor cells.

\section{REFERENCES}

1. Yamada K. Fibronectin and other cell interactive glycoproteins. In: Hay ED, editor. Cell biology of extracellular matrix. New York: Plenum Press; 1989. p. 111-46.

2. Falcone DJ, Salisbury BG. Fibronectin stimulates macrophage uptake of low density lipoprotein-heparin-collagen complexes. Arteriosclerosis 1988;8:263-73.

3. Eriksen HO, Espersen F, Clemmensen I. Opsonic activity of fibronectin in the phagocytosis of Staphylococcus aureus by polymorphonuclear leukocytes. Eur J Clin Microbiol 1984;3: 108-12.

4. Schwarzbauer JE, Tamkun JW, Lemischka IR, Hynes RO. Three different fibronectin mRNAs arise by alternative splicing within the coding region. Cell 1983;35:421-31.

5. Tamkun JW, Schwarzbauer JE, Hynes RO. A single rat fibronectin gene generates three different mRNAs by alternative splicing of a complex exon. Proc Natl Acad Sci U S A 1984;81:5140-4.

6. Kosmehl H, Berndt A, Katenkamp D. Molecular variants of fibronectin and laminin: structure, physiological occurrence and histopathological aspects. Virchows Arch 1996;429:31122 .

7. Hodgkinson CP, Wright MC, Paine AJ. Fibronectin-mediated hepatocyte shape change reprograms cytochrome P450 2C11 gene expression via an integrin-signaled induction of ribonuclease activity. Mol Pharmacol 2000;58:976-81.

8. Odenthal M, Neubauer K, Meyer zum Buschenfelde $\mathrm{KH}$, Ramadori G. Localization and mRNA steady-state level of cellular fibronectin in rat liver undergoing a CC14-induced acute damage or fibrosis. Biochim Biophys Acta 1993;1181: 266-72.

9. Jarnagin WR, Rockey DC, Koteliansky VE, Wang SS, Bissell DM. Expression of variant fibronectins in wound healing: cellular source and biological activity of the EIIIA segment in rat hepatic fibrogenesis. J Cell Biol 1994;127:2037-48.

10. Matsui S, Takahashi T, Oyanagi Y, Takahashi S, Boku S, Takahashi $\mathrm{K}$, et al. Expression, localization and alternative splicing pattern of fibronectin messenger RNA in fibrotic human liver and hepatocellular carcinoma. J Hepatol 1997; 27:843-53.

11. Tavian D, De Petro G, Colombi M, Portolani N, Giulini SM, Gardella R, et al. RT-PCR detection of fibronectin EDA+ and $\mathrm{EDB}+$ mRNA isoforms: molecular markers for hepatocellular carcinoma. Int J Cancer 1994;56:820-5.

12. Fukuda K. Structural alterations in fibronectins secreted from 4 human hepatoma cell lines: demonstration of hepatoma-associated alternative splicing of mRNA precursors. Kurume Med J 1991;38:233-41.

13. Oyama F, Hirohashi S, Shimosato Y, Titani K, Sekiguchi K. Deregulation of alternative splicing of fibronectin pre-mRNA in malignant human liver tumors. J Biol Chem 1989;264: 10331-4.

14. Jagirdar J, Ishak KG, Colombo M, Brambilla C, Paronetto F. Fibronectin patterns in hepatocellular carcinoma and its clinical significance. Cancer 1985;56:1643-8.
15. Ryu S, Jimi S, Takebayashi S. Thyroid carcinoma distinctively expresses intracellular fibronectin in vivo. Cancer Lett 1997; 121:189-93.

16. Svegliati-Baroni G, Ridolfi F, Di Sario A, Saccomanno S, Bendia E, Benedetti A, et al. Intracellular signaling pathways involved in acetaldehyde-induced collagen and fibronectin gene expression in human hepatic stellate cells. Hepatology 2001;33:1130-40.

17. Gratchev A, Guillot P, Hakiy N, Politz O, Orfanos CE, Schledzewski $\mathrm{K}$, et al. Alternatively activated macrophages differentially express fibronectin and its splice variants and the extracellular matrix protein betaIG-H3. Scand J Immunol 2001;53:386-92.

18. Wysocki AB. Fibronectin in acute and chronic wounds. J ET Nurs 1992;19:166-70.

19. Corbett SA, Lee L, Wilson CL, Schwarzbauer JE. Covalent cross-linking of fibronectin to fibrin is required for maximal cell adhesion to a fibronectin-fibrin matrix. J Biol Chem 1997;272:24999-5005.

20. Takayasu H, Horie H, Hiyama E, Matsunaga T, Hayashi Y, Watanabe $\mathrm{Y}$, et al. Frequent deletions and mutations of the beta-catenin gene are associated with overexpression of cyclin D1 and fibronectin and poorly differentiated histology in childhood hepatoblastoma. Clin Cancer Res 2001;7:901-8.

21. Reindel JF, Walsh KM, Toy KA, Bobrowski WF. Spontaneously occurring hepatocellular neoplasia in adolescent cynomolgus monkeys (Macaca fascicularis). Vet Pathol 2000;37: 656-62.

22. Szendroi M, Lapis K, Zalatnai A, Robert L, Labat-Robert J. Appearance of fibronectin in putative preneoplastic lesions and in hepatocellular carcinoma during chemical hepatocarcinogenesis in rats and in human hepatomas. J Exp Pathol 1984;1:189-99.

23. Lynagh GR, Collins RA, Kaiser P. Development and use of monoclonal antibodies to chicken fibronectin to show that the chicken hepatocellular carcinoma cell line, LMH, constitutively expresses fibronectin. Res Vet Sci 2000;68:147-52.

24. Danen EH, Yamada KM. Fibronectin, integrins, and growth control. J Cell Physiol 2001;189:1-13.

25. Iotsova V, Stehelin D. Down-regulation of fibronectin gene expression by the p53 tumor suppressor protein. Cell Growth Differ 1996;7:629-34.

26. Szendroi M, Lapis K. Fibronectin in differential diagnosis of primary hepatomas and carcinoma metastases in the liver. Acta Morphol Hung 1985;33:101-9.

27. Jaskiewicz K, Chasen MR, Robson SC. Differential expression of extracellular matrix proteins and integrins in hepatocellular carcinoma and chronic liver disease. Anticancer Res 1993;13:2229-37.

28. Okushin H, Yamada G, Nagashima H. Immunohistochemical study of fibronectin, lysozyme, and alpha-fetoprotein (AFP) in human hepatocellular carcinoma. Gastroenterol Jpn 1987;22:44-54.

29. Guillouzo A, Belanger L, Beaumont C, Valet JP, Briggs R, Chiu JF. Cellular and subcellular immunolocalization of alphal-fetoprotein and albumin in rat liver. Reevaluation of various experimental conditions. J Histochem Cytochem 1978;26:948-59.

30. Feldmann G, Penaud-Laurencin J, Crassous J, Benhamou JP. Albumin synthesis by human liver cells: its morphological demonstration. Gastroenterology 1972;63:1036-48.

31. Bernuau D, Poliard A, Tournier I, Sala-Trepat J, Feldmann G. All hepatocytes are involved in the expression of the albumin gene in the normal adult rat: a demonstration by in situ hybridization and immunoperoxidase techniques. Cell Biol Int Rep 1985;9:31-42. 